\title{
Nonlinear Transient Behavior of a Sliding System With Frictionally Excited Thermoelastic Instability
}

K. B. Lam

\section{E. Al Bahkali}

\section{J. R. Barber}

University of Michigan, Ann Arbor, Ml 48109
A transient finite element simulation is developed for the two-dimensional thermoelastic contact problem of a stationary layer between two sliding layers, with frictional heat generation. The Petrov-Galerkin algorithm is used to discretize the sliding layers because of the high Peclet numbers involved. The results in the linear, full contact regime were validated by comparison with the analytical predictions of Lee and Barber (1993). After separation occurs, there is a non-monotonic transition to a steady state with the contact regions separated by the same wavelength. During the transition, the migration speed exhibits values lower than those in either the linear regime or the final steady state. When several wavelengths are unstable, the final steady state is generally that corresponding to the longest unstable wavelength, even though other modes have more rapid growth rates in the linear regime. [DOI: 10.1115/1.1353180]

\section{Introduction}

Frictional heating in brakes and clutches causes thermoelastic distortion of the contacting bodies and hence affects the contact pressure distribution. The resulting thermomechanical coupling can cause thermoelastic instability (TEI) if the sliding speed is sufficiently high, leading to non-uniform heating ("hot spots") and low frequency vibration known as "hot judder" [1].

Burton and his coworkers $[2,3]$ showed that the stability of a sliding system can be determined by examining the conditions under which a perturbation in the temperature and stress fields can grow exponentially in time. This method was used by Lee and Barber [4] to examine the stability of a layer sliding between two half-planes - a geometry that can be regarded as an idealization of a brake disk sliding between two friction pads. With realistic automotive friction material properties, they found that the mode with the lowest critical speed was always antisymmetric about the layer mid-plane, corresponding to hot spots alternating on the two sides of the layer. Also, the wavelength of the first eigenmodecorresponding to the hot spot spacing-was approximately 15 times the layer thickness. Yi et al. [5] implemented Burton's method numerically for the limiting case where the friction pads are non-conductors, in which case the perturbation is stationary with respect to the disk. They examined more realistic descriptions of the disk brake geometry, but found that Lee's idealization gives a good approximation to the more exact behavior in most cases.

When both materials are thermal conductors, the exponentially growing perturbation migrates with respect to both bodies. For most braking systems, the conductivity of the disk is very much greater than that of the pad, in which case the predicted migration speed over the disk is relatively slow, but it still has a significant effect on the critical speed, since it reduces the magnitude of the thermoelastic distortion associated with a given perturbation in heat input.

If the system operates above the critical speed, an initial perturbation will eventually grow sufficiently to cause separation to occur somewhere in the nominal contact area, at which point the contact problem becomes non-linear, being governed by the unilateral contact inequalities of non-negative contact pressure and non-negative gap. In most cases, we anticipate that the system will tend to some steady state in which only part of the nominal con-

Contributed by the Tribology Division for publication in the ASME JOURNAL OF TRIBOLOGY. Manuscript received by the Tribology Division August 17, 2000; revised manuscript received January 4, 2001. Associate Editor: M. D. Bryant. tact area remains in contact. Such steady states have been found analytically for some idealized cases where one material is a nonconductor [6], but it remains an open question whether they exist for systems of two conductors and if so, whether the resulting contact area migrates over the surface of both bodies or is stationary with respect to one of them. Experimental observations of hot spots in automotive brake disks [7] suggest that migration is very slow, if it occurs at all.

In the present paper, we shall investigate these questions by using numerical simulation. We discretize both the thermoelastic contact problem and the transient heat conduction problem using the finite element method. Early attempts at numerical simulation of TEI in a disk brake were made by Kennedy and Ling [8]. Azarkhin and Barber $[9,10]$ developed solutions for the transient thermoelastic Hertz problem, using Green's function and Fourier transform techniques. Zagrodzki [11] developed a transient thermoelastic simulation for axisymmetric deformation of a multidisk clutch. All of these papers were restricted to cases where there was no migration of the thermoelastic disturbance in the sliding direction. In Kennedy's and Zagrodzki's analyses, the behavior was assumed to be axisymmetric, making the sliding outof-plane, whereas in that of Azarkhin, one of the materials was assumed to be a non-conductor. Simulation is more challenging when migration is present, since at least one of the materials then flows through the frame of reference and the corresponding Peclet numbers are generally large.

\section{Model}

2.1 Geometry. The sliding system is represented by the two-dimensional model shown in Fig. 1. Each component of the system is elastic and thermally conducting. The two external blocks 1 and 3 move in-the-plane with respect to the central layer 2 at speed $V$. Blocks 1 and 3 represent the same material while the central layer 2-a dissimilar material. Solutions of the thermoelastic problem are assumed to be cyclically symmetric in the sliding direction $x$ with wavelength $L=C / n$, where $C$ represents the circumference of the disk and $n$ is an integer.

2.2 Heat Transfer. A common reference system $O x y$ for the whole model is used. In general, each component of the model can move in $x$-direction with respect to this system. It is convenient, however, to fix the system to a selected component. The selection will be based on the expected behavior of pressure perturbation. It is known that for both mating materials being conductors, the perturbation migrates in the sliding direction with 


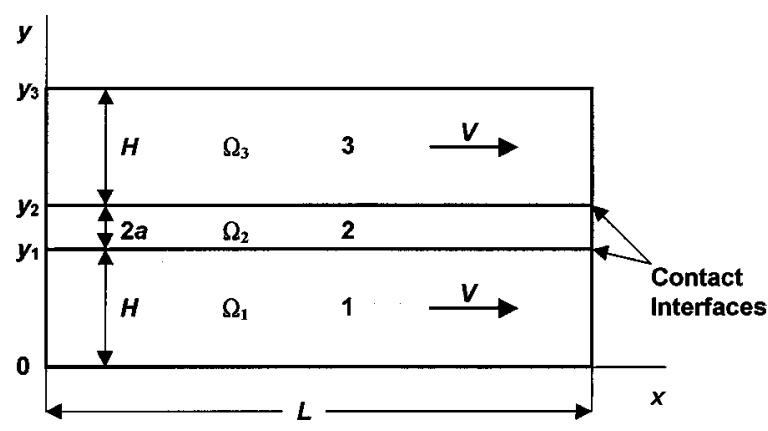

Fig. 1 Schematic of sliding system

respect to each of them. If the materials are dissimilar, the speed of migration with respect to the material of higher thermal conductivity is small comparing to the sliding speed [4]. On the other hand, the speed at which the perturbation moves with respect to the reference system affects the time step in the numerical solution of transient thermoelastic problem, and higher speed generally demands a shorter time step. Therefore, it is advantageous for numerical efficiency that the reference system is fixed to the better conductor. In the model shown in Fig. 1, the layer 2 represents ferrous material, which is a good conductor, while the blocks 1 and 3 represent friction material, which is usually a poor conductor. Consequently the reference system is chosen to be fixed to the layer 2 .

Note that only components that are continuous in the $x$-direction can be treated as moving. In a sliding system with contact surfaces that are not coextensive in the sliding direction, like an automotive disk brake, the geometry would dictate that the reference system be fixed to the pads regardless of relative conductivity of materials.

The heat transport in moving components 1 and 3, Fig. 1, is described by a conduction-convection equation of the form

$$
K_{i} \nabla^{2} T_{i}=c_{p i} \rho_{i}\left(\frac{\partial T_{i}}{\partial t}+V \frac{\partial T_{i}}{\partial x}\right) \text { in } \Omega_{i}, \quad i=1,3 .
$$

Here, $c_{p}, \rho$, and $K$ denote specific heat, density, and conductivity, respectively; $V$ is sliding speed and the operator $\nabla=\partial / \partial x+\partial / \partial y$. The properties of materials 1 and 3 are the same, i.e., $c_{p 3}=c_{p 1}$, $\rho_{3}=\rho_{1}$, and $K_{3}=K_{1}$.

The heat conduction in the layer 2 is described by the equation

$$
K_{2} \nabla^{2} T_{2}=c_{p 2} \rho_{2} \frac{\partial T_{2}}{\partial t} \text { in } \Omega_{2} .
$$

Heat generation at the contact interfaces $y=y_{i}(i=1,2)$ is defined by

$$
q_{i}=f V p_{i},
$$

where $f$ is friction coefficient, and $p_{i}$ is contact pressure at the interface. The interfacial boundary conditions on $y=y_{i}$ depend on the status of mechanical contact. If the surfaces are in contact, i.e., if the clearance $\Delta u_{y}=0$, they are given implicitly by the energy conservation condition

$$
\left.K_{i} \frac{\partial T_{i}}{\partial y}\right|_{y=y_{i}}-\left.K_{i+1} \frac{\partial T_{i+1}}{\partial y}\right|_{y=y_{i}}=q_{i}, \quad i=1,2,
$$

and the temperature continuity condition

$$
\left.T_{i}\right|_{y=y_{i}}=\left.T_{i+1}\right|_{y=y_{i}}=0, \quad i=1,2 .
$$

If $\Delta u_{y}=0$, the separated surfaces are treated as adiabatic

$$
\left.\frac{\partial T_{i}}{\partial y}\right|_{y=y_{i}}=\left.\frac{\partial T_{i+1}}{\partial y}\right|_{y=y_{i}}=0, \quad i=1,2 .
$$

Additionally, on the boundaries $x=0$ and $x=L$ the following conditions imposing cyclic symmetry are enforced

$$
\left.T_{i}\right|_{x=0}=\left.T_{i}\right|_{x=L}, \quad i=1,2,3 .
$$

2.3 Method of Solving of Heat Transfer Problem. Equation (1) has a dual, conductive-convective, nature. It implies serious difficulties with numerical solution by either finite element or finite difference methods. The standard Galerkin finite element algorithm, which is of central-difference type, is suitable for discretizing the diffusive term in Eq. (1), but it does not provide an adequate discretization of the convective term $V \partial T / \partial x$. If this term is significant, namely if the mesh Peclet number $\gamma$ $=V h c_{p} \rho / K$ is greater than 2 , where $h$ is a characteristic element length, the algorithm exhibits excessive numerical oscillations and provides spurious solutions [12]. Usually $\gamma>100$ for typical friction clutch/brake models. A scheme that is consistent with the nature of the convective term, is the one of backward-difference type. To handle convection dominated problems, a modified finite element algorithm, called Petrov-Galerkin, has been developed $[12,13,14]$. The algorithm uses an upwinding approach, which basically consists in replacing the central-difference scheme with a backward-difference scheme for both convective and diffusive terms. This is obtained by using non-symmetric weighting functions in finite element formulation, while the shape functions are the same as in conventional Galerkin formulation.

Simulation of a time-dependent convection dominated problem involves an additional difficulty. Numerical dispersion occurs, producing phase errors, varying with the wavelength. $\mathrm{Yu}$ and Heinrich $[13,14]$ show that this effect can be controlled by using specially designed time-space finite elements with weighting functions linear in space and quadratic in time.

In this work we use a commercial finite element package Abaqus/HKS [15], equipped with the Petrov-Galerkin algorithm based on $[13,14]$. Before solving the actual problem, we broadly tested the algorithm by solving a trial thermoelastic contact problem for which analytical solution is known.

2.4 Elastic Contact. A standard static elastic contact formulation is used which imposes continuity of normal displacements $u_{y}$ across the contact interfaces if the contact condition is satisfied.

If $\left(u_{y}\right)_{i}$ denotes displacement component in $i$ th region (Fig. 1), the boundary conditions on the edges $y=0$ and $y=y_{3}$ have the form

$$
\left.\left(u_{y}\right)_{1}\right|_{y=0}=0 \text { and }\left.\left(u_{y}\right)_{3}\right|_{y=y_{3}}=\text { const. }
$$

with the total external force $F_{y}$ in the $y$ direction being controlled. On the edges $x=0$ and $x=L$, cyclic symmetry conditions

$$
\left.\left(u_{x}\right)_{i}\right|_{x=0}=\left.\left(u_{x}\right)_{i}\right|_{x=L} \text { and }\left.\left(u_{y}\right)_{i}\right|_{x=0}=\left.\left(u_{y}\right)_{i}\right|_{x=L}, \quad i=1,2,3
$$

are imposed.

The elastic contact problem is also solved by the finite element method using Abaqus code.

2.5 Sequential Numerical Simulation of Thermoelastic Contact Problem. In the simulation procedure the thermal problem (Section 2.2) and elastic contact problem (Section 2.4) are solved sequentially in time.

2.5.1 Finite Element Mesh. A typical practical combination of mating materials in a brake or a clutch is that with dissimilar materials. In this case the perturbation in contact pressure/ temperature moves at relatively high speed, close to $V$, with respect to the poor conductor. A particular point on the surface of this material experiences temperature oscillations at a frequency $\omega$ approximately equal to $2 \pi V / L$, where $L$ is the length of the wave. The character of the temperature distribution in the poor conductor in the $y$ direction normal to the surface can be estimated by solving a one-dimensional transient heat conduction problem in 


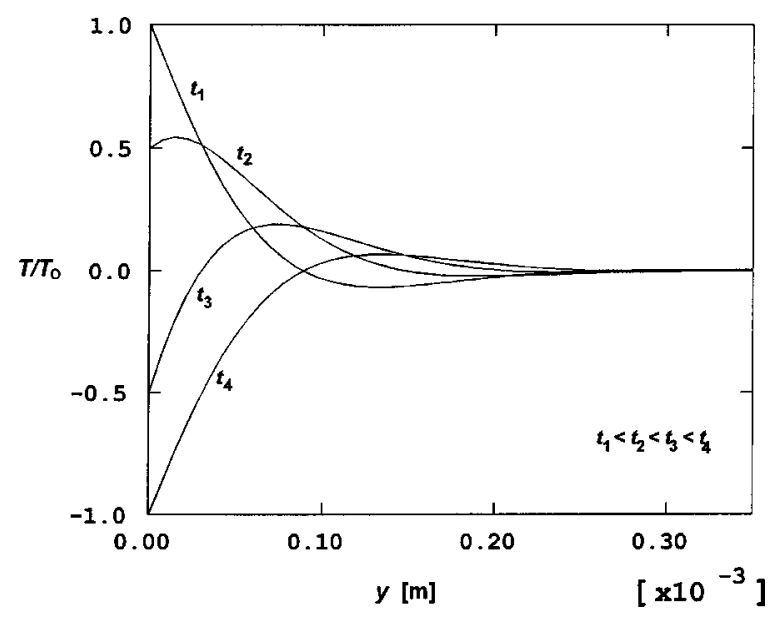

Fig. 2 Character of temperature distribution in a skin layer of poor conductor

half-infinite domain with an oscillatory boundary condition. For the boundary condition $\left.T(t)\right|_{y=0}=T_{s} \cos \omega t$, where $T_{s}$ is the amplitude of surface temperature, the solution has the form [16]

$$
T(t, y)=T_{s} \exp (-\psi y) \cos (\omega t-\psi y),
$$

where $\psi=\sqrt{\omega / 2 k}$ is both the spatial frequency and the spatial damping factor, and $k=K / c_{p} \rho$ is thermal diffusivity. For the practical range of parameters $\omega$ and $k$, the parameter $\psi$ takes high values, implying that the temperature distribution in the poor conductor in the direction normal to the surface is represented by very short wavelength and is strongly damped. Hence, a noticeable temperature variation occurs only in a thin skin at the surface. Figure 2 shows the solution (10) for an example set of realistic parameters $\left(V=5 \mathrm{~m} / \mathrm{s}, L=0.05 \mathrm{~m}, k=2 \times 10^{-6} \mathrm{~m}^{2} \mathrm{~s}^{-1}\right)$, for which $\psi=1.7725 \cdot 10^{4} \mathrm{~m}^{-1}$ and the corresponding wavelength $\lambda$ $=2 \pi / \psi=0.35 \mathrm{~mm}$.

The finite element mesh must be designed to be capable of reproducing this strong variation of temperature in the skin layer of friction material. At the same time the mesh, which is common for thermal and elastic problems, must adequately represent the mechanical behavior of the system. In particular, a meaningful strain variation is expected also at locations distant from the contact interface, and, therefore, the modeled area cannot be confined to the skin only. In the good conductor, with respect to which the perturbation migrates slowly, much smaller temperature gradients in the $y$-direction are expected. Consequently, much larger element size $h_{y}$ is used in this area with a uniform mesh, as shown in Fig. 3.

The mesh in sliding direction $x$ is uniform in each component. The smallest wavelength captured by the discrete model of convective problem is $2 h_{x}$ [17]. However, the accuracy of wavelengths represented by small number of elements is poor and therefore a finer mesh than that resulting from this condition should be used. Note that in nonlinear range of operation of our system, with contact separation, the solution is no longer sinusoidal in the sliding direction and it contains also higher Fourier components. In this study we use a mesh in which the wavelength $L$ of the lowest mode is divided into 50 elements.

We use quadrilateral bilinear elements. The skin layer of the poor conductor of thickness about $0.75 \lambda$ is divided into several elements in $y$-direction with very small size $h_{y}$, Fig. 3 . The elements in this region have aspect ratio $h_{x} / h_{y} \gg 1$.

2.5.2 Time Step. The Petrov-Galerkin finite element algorithm for solving convection dominated problems may include control of numerical dispersion. In this case the algorithm is conditionally stable with stability condition of the form $\mathrm{Cu} \leqslant 1$, where Courant number $\mathrm{Cu}=V \Delta t / h_{x}$ [13]. For given mesh size in flow

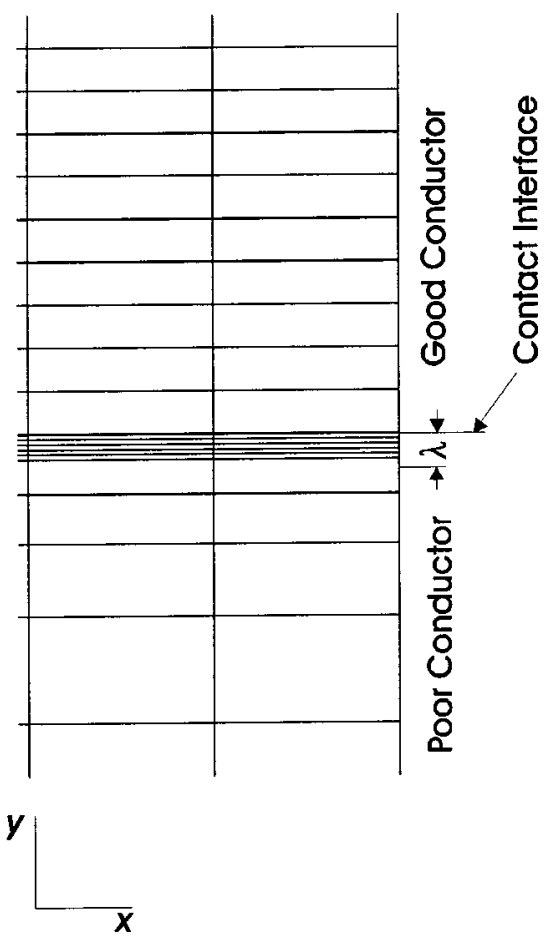

Fig. 3 Detail of finite element mesh

direction $h_{x}$ and flow speed $V$, this condition imposes an upper limit on the time step $\Delta t$. The algorithm without dispersion control is unconditionally stable. However our tests indicate that the accuracy that it provides for $\mathrm{Cu}$ substantially greater than 1 is not satisfactory. On the other hand, for $\mathrm{Cu} \leqslant 1$ both algorithms gave practically the same results for the range of wavelengths under consideration. Hence, we chose the time step that satisfies $\mathrm{Cu} \leqslant 1$, without preferring any of the two versions of the algorithm.

In the component 2, Fig. 1, pure conduction occurs. Also, in components 1 and 3 the heat is transported in direction $y$ by conduction only. It is known that a short time step in finite element algorithms for conduction problems can produce numerical oscillations [15]. The requirement for the time step dictated by this effect would generally be in conflict with the upper limit on the time step discussed above. Therefore, it is crucial to use a discretization scheme that provides oscillation-free solutions even for very short time step. This feature is exhibited by the first order finite elements with lumped heat capacity matrix [17]. Although the upwinding modifies the structure of the heat capacity matrix, introducing out-of-diagonal elements, the lumped structure in the cross-wind direction is preserved, and our tests showed lack of noticeable oscillation of solution with the mesh size and time step used.

The condition $\mathrm{Cu} \leqslant 1$ enforces in practice a very short time step. For instance, $V=5 \mathrm{~m} / \mathrm{s}, h_{x}=1 \cdot 10^{-3} \mathrm{~m}$ requires that $\Delta t$ $\leqslant 2 \cdot 10^{-4}$ s. Note that the elastic properties of the mating materials usually differ considerably. While the thermal expansion coefficients of the materials are of the same of magnitude, the modulus of elasticity of the friction material is typically orders of magnitude lower than that of the mating ferrous material. Consequently, the solution of elastic contact problem is mostly governed by the temperature field in the ferrous material. Since the perturbation migrates slowly with respect to this component, the evolution of the thermoelastic field there is relatively slow and the characteristic time of this process is much longer than the time step $\Delta t$ required for thermal analysis in the whole system. Thus, it is reasonable to use in the elastic analysis a time step $\Delta t_{e}=N$ $\cdot \Delta t$, where $N$ is an integer. Practical values of $N$ were determined 
by trial simulations for particular processes characterized by specific growth rate and migration speed. Better than 1.5 percent accuracy in these quantities was achieved with values of $N$ in the range 4 to 10 , depending on the sliding speed.

In the thermal analysis, the heat flux at time $t+n \Delta t$, where $t$ is the time at which the solution is known and $n=1, \ldots, N$, is approximated by $q_{i}^{t+n \Delta t}=f V p^{t}$ where $p^{t}$ is the contact pressure at time $t$. Then, after calculating $T^{t+N \Delta t}$, the elastic contact problem at the time $t+N \Delta t$ is solved.

\section{Validation of Finite Element Model}

3.1 Growth Rate and Migration Speed of Perturbation. In order to verify the simulation model against Lee and Barber's analytical model [4], a transient solution corresponding to the single eigenmode is simulated. Basing on its behavior, the growth rate is estimated. In fact, the simulated thermoelastic process includes both the underlying process, corresponding to the constant part of the contact pressure, and the overimposed perturbation. Note that the underlying process is irrelevant to the stability as long as the system is linear. The pressure on the contact interface is expected to vary according to the equation

$$
p(x, t)=p_{m}+p_{a 0} \cos [2 \pi(x+c t) / L] \exp (b t),
$$

where $p_{m}$ is mean pressure, controlled by external force applied to the system and the second term represents the pressure perturbation; $p_{a 0}$ is its initial amplitude, and $b$ is the growth rate. The mean pressure remains constant during the process. Denoting the amplitude of the variable part of the pressure by $p_{a}(t)$, we can write

$$
\ln \left[p_{a}(t) / p_{m}\right]=\ln \left(p_{a 0} / p_{m}\right)+b t .
$$

In the simulation, a particular mode of perturbation is triggered by perturbing the contact pressure in the initial step of thermal analysis. This initial condition reflects only the general pattern of the eigenmode of the interest and the actual mode forms gradually during the initial stage of the simulation. After that initial phase, we observed that the pressure varies according to the formula (12), i.e., $\left.\ln \left[p_{a}(t) / p_{m}\right)\right]$ varies linearly in time.

Figure 4 shows pressure distribution at a series of instants obtained for the system parameters specified in Table 1 and wavelength $L=0.025 \mathrm{~m}$, sliding speed $V=3.6 \mathrm{~m} / \mathrm{s}$. Fig. 5 shows variation of $\ln \left[p_{a}(t) / p_{m}\right]$ and the migration of the perturbation in sliding direction for two sliding speeds of $2.0 \mathrm{~m} / \mathrm{s}$ and $3.6 \mathrm{~m} / \mathrm{s}$. The slope of $\left.\ln \left[p_{a}(t) / p_{m}\right)\right]$ for the speed of $3.6 \mathrm{~m} / \mathrm{s}$ is positive, thus the solution is unstable. For $V=2 \mathrm{~m} / \mathrm{s}$ the solution is stable.

Figure 6 shows the part of the temperature distribution corresponding to the perturbation only, extracted from the complete solution. This temperature distribution corresponds to the stage of the process with steady-state growth, ruled by the Eq. (12), hence it represents the dominant eigenmode.

The growth rate is determined from the equation

$$
b=\ln \left[p_{a}(t+\Delta t) / p_{a}(t)\right] / \Delta t .
$$

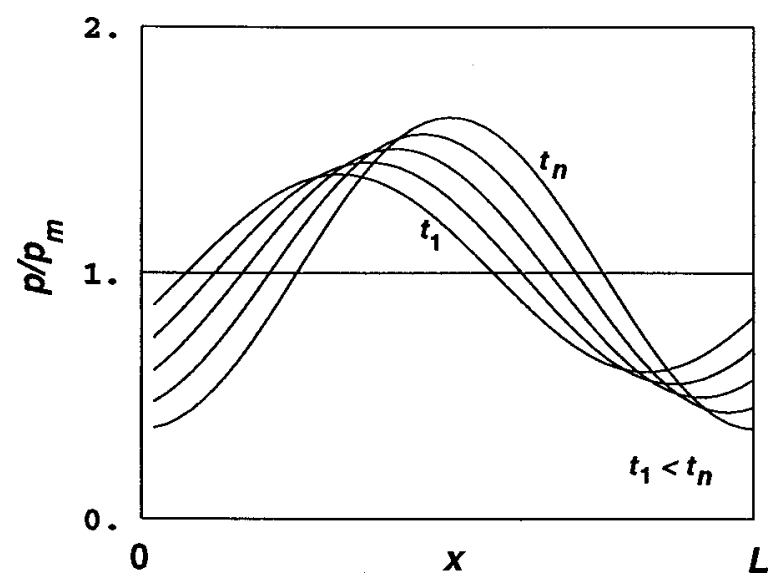

Fig. 4 Contact pressure distribution at a series of instants during the stage with full contact

3.2 Comparison Against Lee and Barber Model. A series of simulations were run to validate the finite element model against Lee and Barber's [4] analytical model of a layer sliding between two half-planes. The Lee and Barber solutions for the critical sliding speed and migration speed of perturbation are reproduced in Figs. 7 and 8, respectively, using friction coefficient $f=0.2$ and the properties given in Table 1 . These properties, except for the Poisson's ratios, are representative of steel and some friction materials used in clutch and brake applications. In the validation tests we neglected the mechanical effect of shear tractions in order to reduce computation time, whereas these effects are included in Lee's model. This approximation is exact if $\nu$ $=0.5$ for both materials [18] and we therefore chose values of $\nu$ close to 0.5 in order to reduce the effect of this discrepancy between the models. For other values of $\nu$, shear effects may increase or decrease the critical speed depending on the material properties, but the effect is generally quite modest when the conductivities of the sliding bodies are very different [18].

The wavenumber of the perturbation $m$ and the dimensionless wavenumber $A$ are defined

$$
m=2 \pi / L, \quad A=m a,
$$

where $a$ is the half-thickness of the central layer. The dimensionless critical and migration speeds are defined by

$$
V_{c r}^{*}=V_{c r} a / k_{2}, \quad c^{*}=c a / k_{2},
$$

respectively, where $V_{c r}$ is the critical speed (i.e., the speed at which the perturbation growth rate is zero), $c$ is the migration speed, and $k_{2}$ is the thermal diffusivity of the central layer.

In performing the validation, a set of discrete wavenumbers from $A=0.245$ to 1.0 was selected, with a particular concentration near the minimum of the curve. $A=0.245$ represented the lowest wavenumber at which higher-order perturbation modes remained

Table 1 Properties and geometry of blocks 1 and 3 and central layer 2

\begin{tabular}{|l|c|c|c|c|c|c|}
\hline & $\begin{array}{c}\text { Conductivity } K \\
(\mathrm{~W} / \mathrm{m} \cdot \mathrm{K})\end{array}$ & $\begin{array}{c}\text { Thermal } \\
\text { Diffusivity } k \\
\left(\times 10^{-6} \mathrm{~m} / \mathrm{s}^{2}\right)\end{array}$ & $\begin{array}{c}\text { Modulus of } \\
\text { Elasticity } E \\
\left(\times 10^{9} \mathrm{~N} / \mathrm{m}^{2}\right)\end{array}$ & $\begin{array}{c}\text { Poisson's } \\
\text { Ratio } v\end{array}$ & $\begin{array}{c}\text { Thermal } \\
\text { Expansion } \\
\text { Coefficient } \alpha \\
\left(\times 10^{-6} \mathrm{~K}^{-1}\right)\end{array}$ & $\begin{array}{c}\text { Height } H \\
(\mathrm{~m})\end{array}$ \\
\hline $\begin{array}{l}\text { External } \\
\text { Blocks 1 \& 3 }\end{array}$ & 1.0 & 4.167 & 3.0 & 0.494 & 12.0 & 0.050 \\
\hline $\begin{array}{l}\text { Central Layer } \\
2\end{array}$ & 42.0 & 11.91 & 210.0 & 0.494 & 12.0 & 0.010 \\
\hline
\end{tabular}



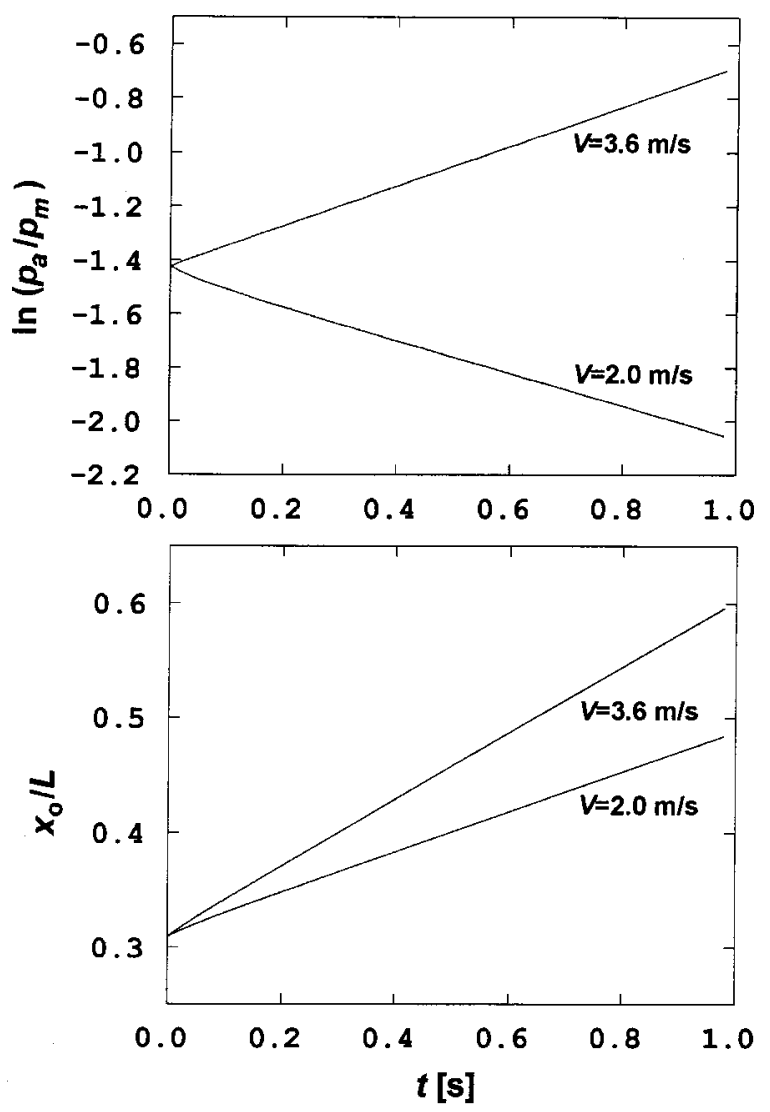

Fig. 5 Change in amplitude and location of pressure perturbation: $p_{a}$-amplitude of pressure perturbation; $p_{m}$-mean pressure; $x_{0}$-location of maximum pressure along $x$-axis

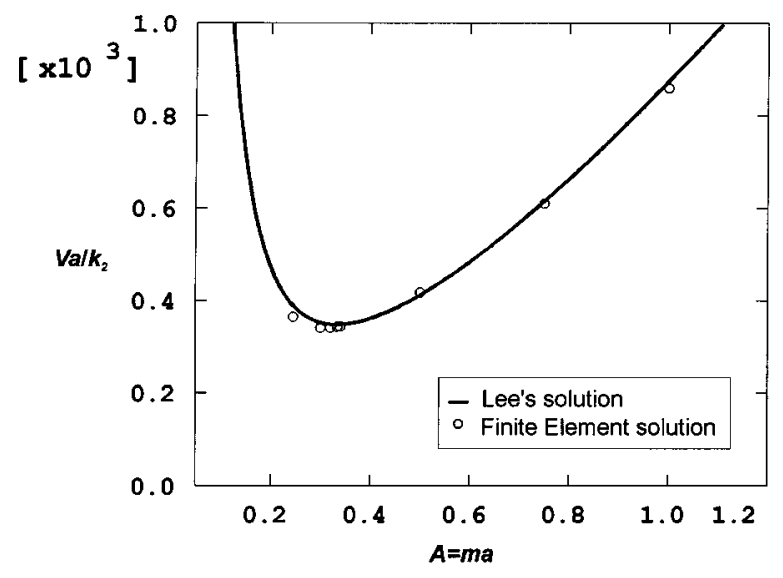

Fig. 7 Dimensionless critical speed determined by Lee and Barber's analytical model and estimated by finite element model

stable according to Lee's solution. To attain each discrete wavenumber, the length of the FE model was adjusted according to Eqs. (14) $(L=2 \pi a / A)$, and one wave of an initial pressure perturbation was imposed. The thickness of the external blocks was kept constant at $H=0.050 \mathrm{~m}$. Simulations were then run at each discrete $A$ with sliding speed 5 percent above and below the Lee critical $V_{c r}^{*}$ for times sufficient for the perturbation growth rates to converge.

As expected, it was observed that the growth rates were slightly positive and negative, respectively, for the speeds 5 percent above and below $V_{c r}^{*}$. Using linear interpolation, the speed at which the FE model would be estimated to produce zero growth rate was found for each discrete $A$. These results are compared with Lee's curve in Fig. 7. It can be seen that the FE model generally slightly

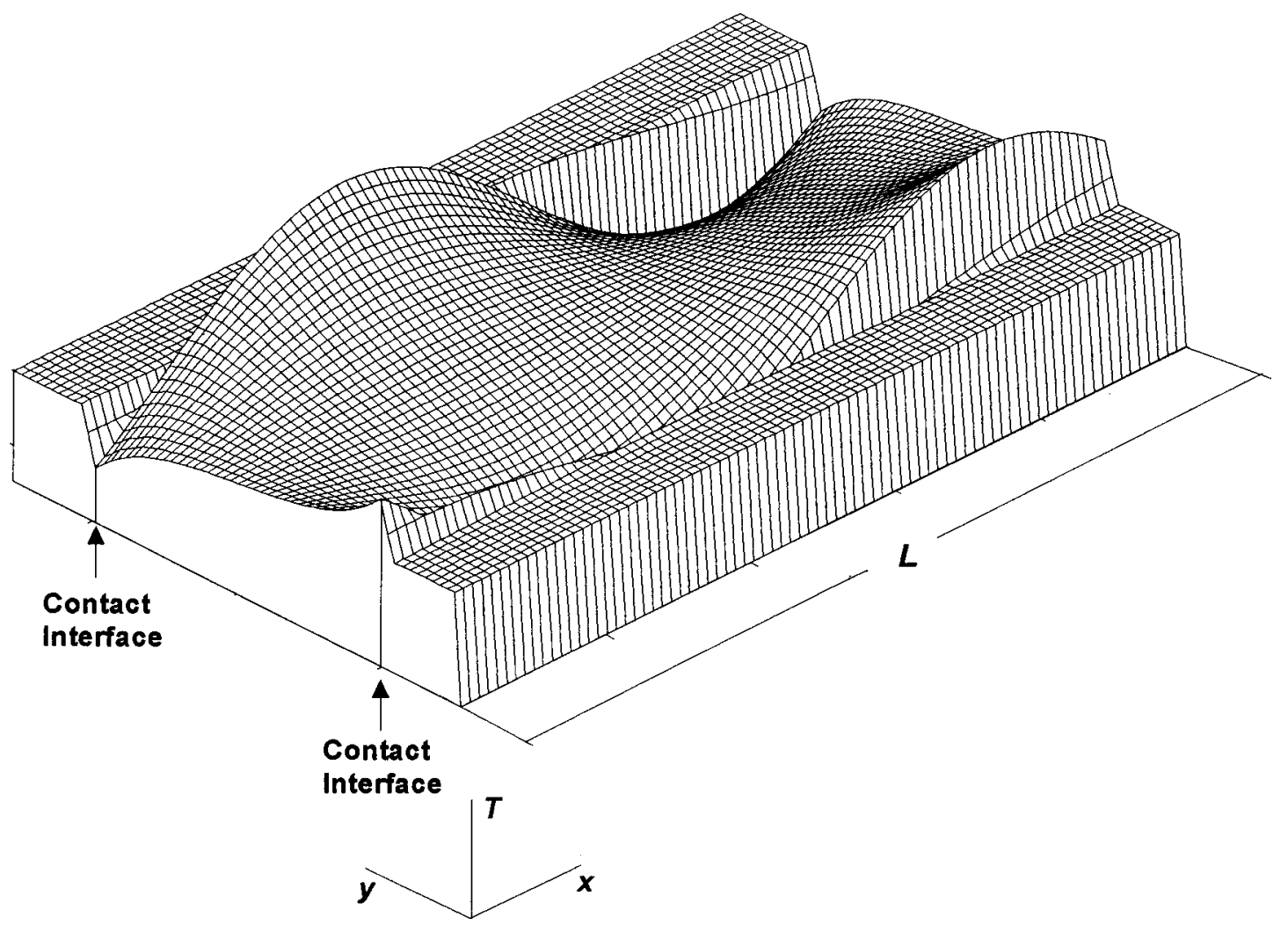

Fig. 6 Temperature distribution representing dominant eigenmode 


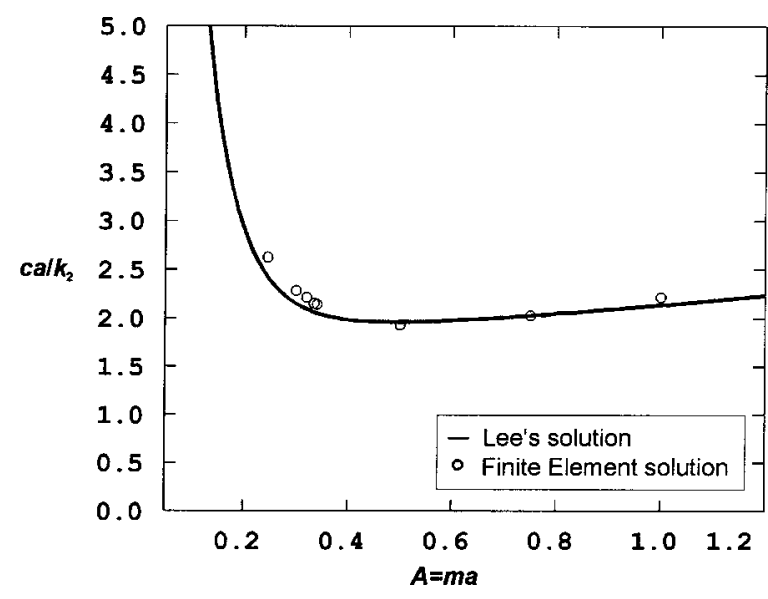

Fig. 8 Dimensionless migration speed determined by Lee and Barber's analytical model and estimated by finite element model

underestimates the critical speeds compared to Lee's model. The differences were for most of the simulated points less than 4 percent. However, for the lowest discrete wavenumber $A=0.245$, the difference is about 7 percent. At this wavenumber, $L=0.13 \mathrm{~m}$, so that the ratio $H / L=0.38$. Yi et al. [5] showed that ratios of $H / L$ $<0.5$ yielded reduced critical speeds relative to Lee's model $(H / L \rightarrow \infty)$, which explains the bigger discrepancy between Lee's and our model at low $A$. The FEM migration speed $c^{*}$ at zero growth rate was also estimated at each discrete $A$ and is plotted
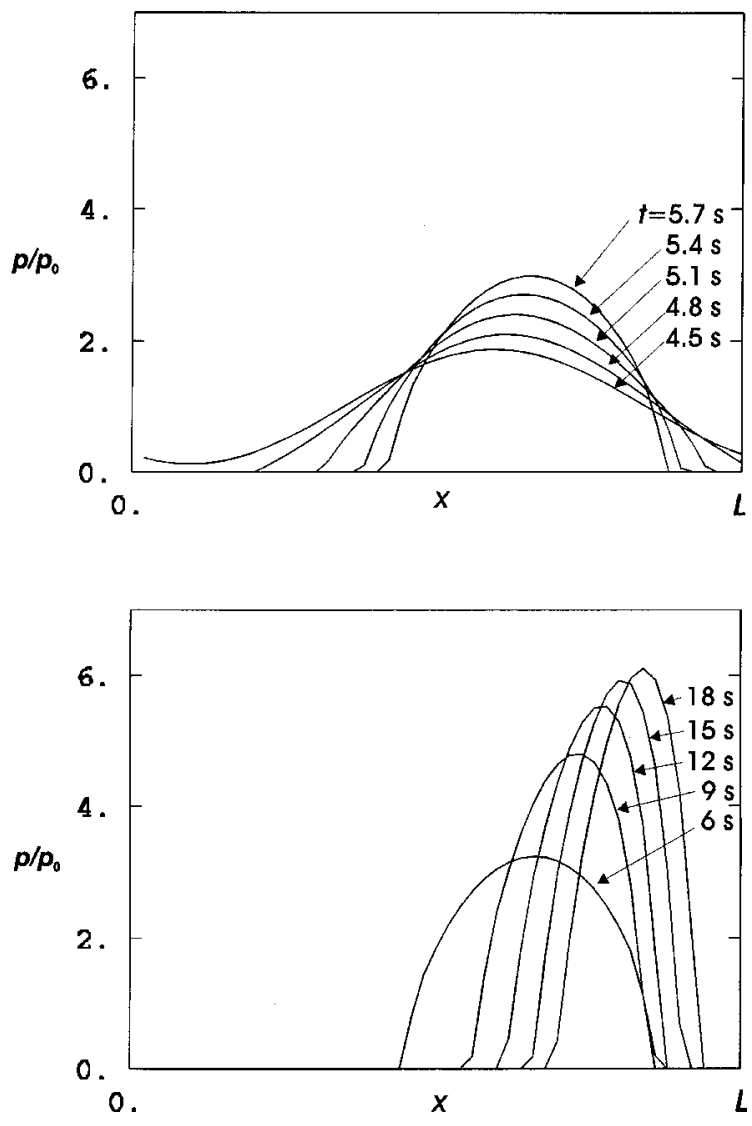

Fig. 9 Contact pressure distribution at a series of instants during the stage with contact separation
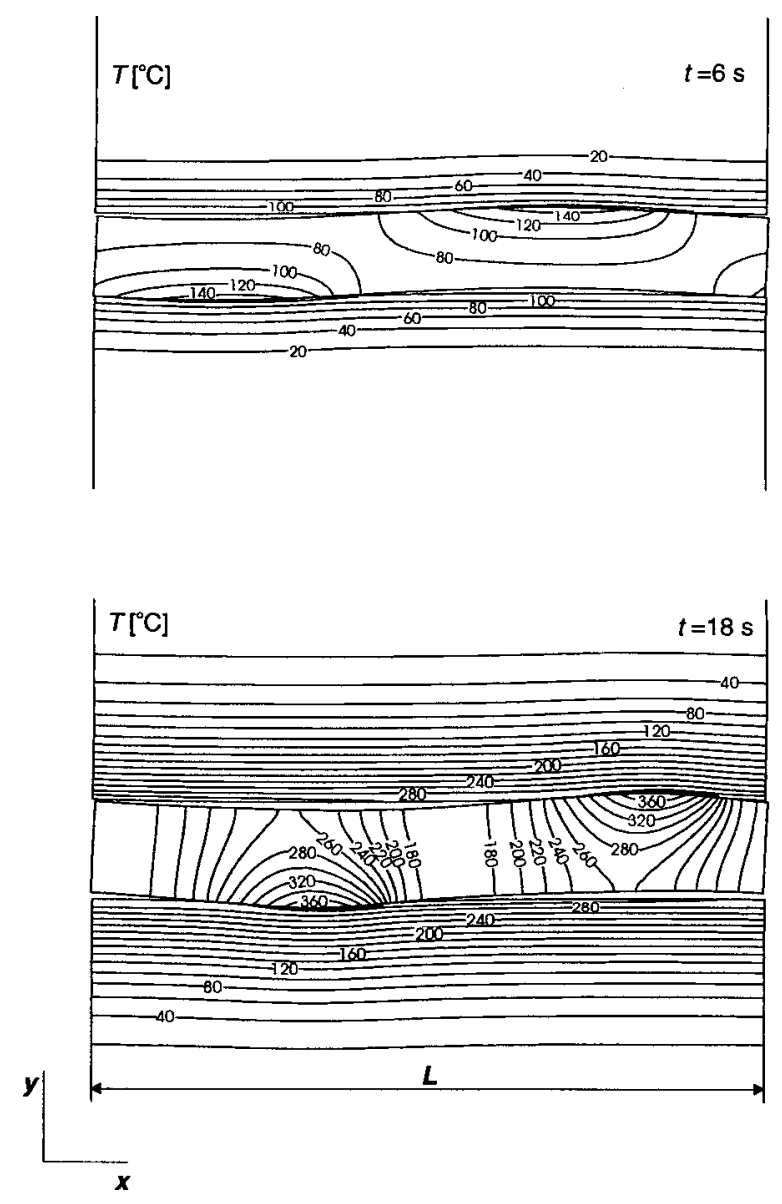

Fig. 10 Temperature contours at different instants

against the Lee solution in Fig. 8. Again, the agreement between the two models is quite good-within 5 percent at each discrete $A$, except at $A=0.245$, where it was 7 percent.

\section{Transient Solution}

4.1 Solution with Single Mode Unstable. The minimum critical speed corresponds to wavenumber $A=0.334$ (the dimensional wavelength is $L=0.09325 \mathrm{~m}$ ), see Fig. 7. We simulated the thermoelastic process for this wavelength for a series of sliding speeds: $1.25 V_{c r}, 1.5 V_{c r}$, and $2.0 V_{c r}$.

Figure 9 shows the contact pressure distribution at a series of instants for speed $V=1.5 V_{c r}$. Separation of contact starts at $t$ $=4.7 \mathrm{~s}$ and is followed by rapid contraction of the contact area and a corresponding increase in maximum contact pressure. Figure 10 shows the temperature fields at $t=6 \mathrm{~s}$, shortly after separation begins, and at $t=18 \mathrm{~s}$. The corresponding temperature profiles along the upper contact interface are shown in Fig. 11. The pattern of temperature distribution changes considerably during the separation regime. At $t=6 \mathrm{~s}$, the mode retains approximately the antisymmetric character of the full contact solution, whereas the $t=18 \mathrm{~s}$, a second local maximum of temperature is developed on the layer surface. This maximum is opposite to the hot spot on the other sliding surface and results from heat penetration across the layer.

Figures 12 and 13 show the evolution of the maximum contact pressure $p_{\max }$, contact length $L_{c}$ and migration speed $c_{s}$ during the transient process for $V=1.5 V_{c r}$. Figure 12 shows the early part of the process $(0 \leqslant t \leqslant 16 \mathrm{~s})$. During the initial full contact phase, the perturbation in contact pressure $\left(p_{\max }-p_{m}\right)$ grows exponentially and the migration speed is constant, as predicted by Lee's theory. After separation begins, the migration speed de- 

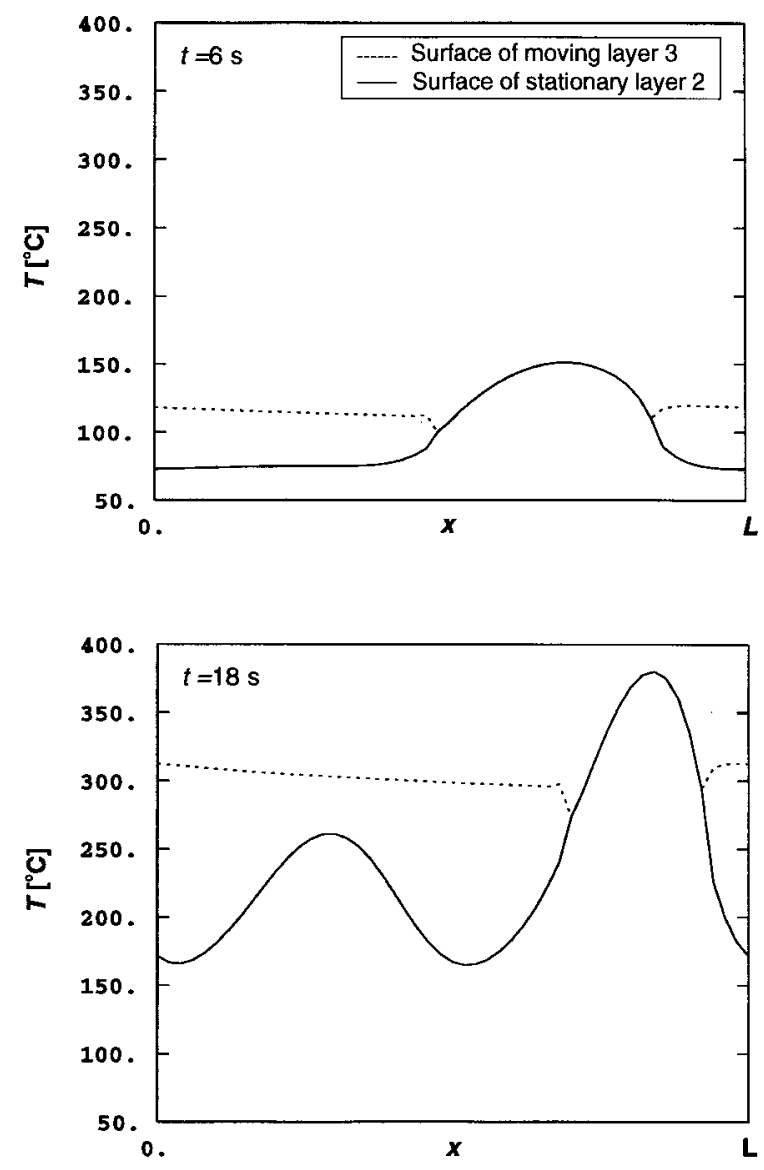

Fig. 11 Temperature distribution along contact surfaces at different instants

creases significantly, as does the rate of increase of the maximum contact pressure. However, the maximum contact pressure continues to grow and it reaches a very high value. The process appears to be tending monotonically towards a steady state, but this is illusory, as seen from Fig. 13, which extends the results out to $t$ $=450 \mathrm{~s}$. In fact, the transient process overshoots the steady state and there is a gradual return to a larger contact area and lower maximum contact pressure. The form of the contact pressure distribution evolves during this process. Figure 14 shows the contact pressure at two instants $t=6.3 \mathrm{~s}$ and $t=420 \mathrm{~s}$ before and after the extremum point respectively, chosen so as to have approximately the same value of $p_{\max }$. In this plot, one of the curves was moved in the $x$-direction in order to make the comparison easier. Notice that the curves have different shapes. The pressure distribution at $t=420 \mathrm{~s}$, close to the final steady state, is significantly asymmetric. This evolution of the form of the contact pressure distribution is associated with a striking change in the migration speed $c_{s}$, which falls dramatically during the initial separation phase but then returns to a value not far below that in the full contact phase. Large numbers of time steps are required for these long time simulations, but extrapolation at large time using exponential functions suggests that the final migration speed will be 89 percent of that in the full contact regime, in contrast to a minimum of 14.5 percent at about $t=20 \mathrm{~s}$. The corresponding extrapolated values for the maximum contact pressure and the contact length $p_{\max } / p_{m}=3.4$ and $L_{c} / L=0.44$.

4.2 Solution with Multiple Unstable Modes. The most common sign of thermoelastic instability in clutches and brakes is the occurrence of focal discolorations on the surface of the steel or cast iron disks after operation $[19,20]$. These often have a fairly regular pattern, reflecting a single eigenmode [20], but in other
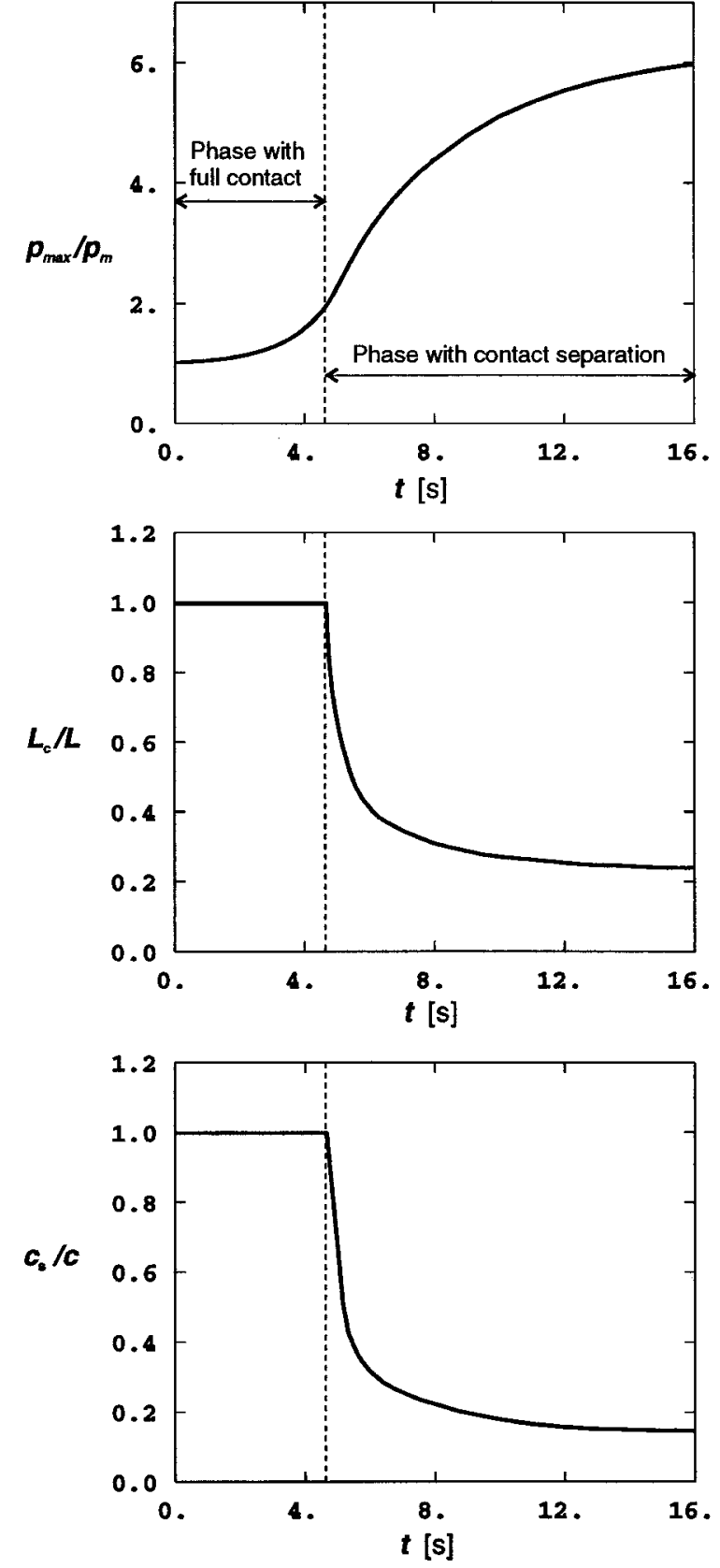

Fig. 12 Maximum contact pressure, contact length and migration speed: $p_{\max }, p_{m}$-maximum and mean contact pressure, respectively; $L_{c}$-contact length; $L$-length of the interface; $c_{s}$-migration speed; $c$-migration speed in the full contact phase.

cases less regular patterns are obtained. These can result from a sequential occurrence of hot spots in a series of applications [20], from geometric imperfections of the surfaces or from the simultaneous excitation of several different modes. In the present section we shall explore the development of hot spots when more than one mode is unstable.

For a periodic system of finite length $L$, the wavelength must be an integer submultiple of $L$, and, hence, the wavenumber can take only the values $m=2 n \pi / L$, where $n$ is an integer. This is illustrated in Fig. 15, which also reproduces Lee's solution for the critical speed as a function of dimensionless wavenumber $m a$. The length $L$ was selected so that the critical speed of the mode of wavelength $L$ is higher than that with wavelength $L / 2$. Simulations were performed at the three speeds labeled $V_{1}, V_{2}, V_{3}$, in 

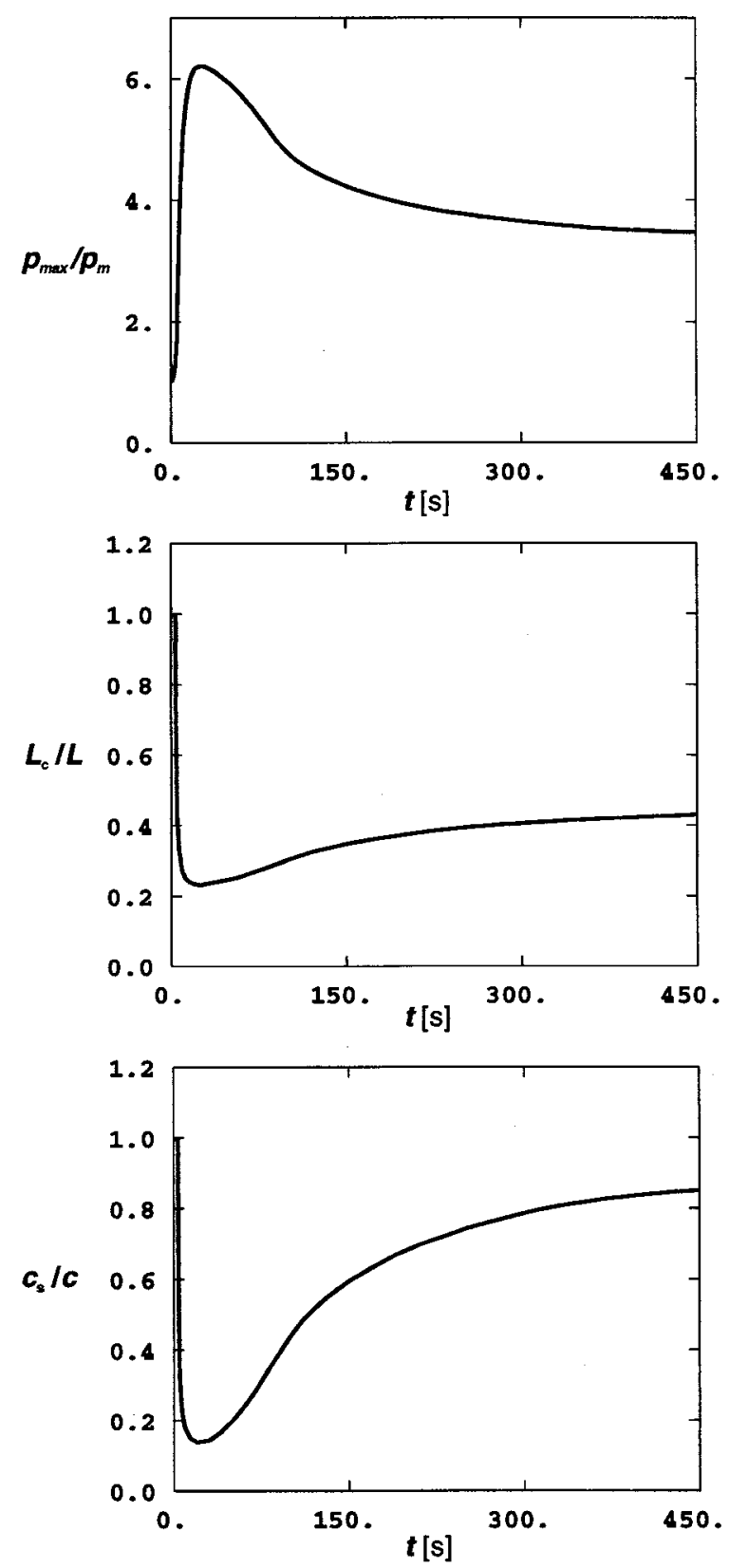

Fig. 13 Maximum contact pressure, contact length and migration speed: $p_{\max }, p_{m}$-maximum and mean contact pressure, respectively; $L_{c}$-contact length; $L$-length of the interface; $c_{s}$-migration speed; $c$-migration speed in the full contact phase

Fig. 15. Speed $V_{1}$ is slightly above the critical speed for the first mode of wavelength $L$, but significantly above it for the second mode of wavelength $L / 2$. In addition, the amplitude of the initial perturbation for the second mode was chosen to be ten times that for the first mode. The resulting evolution of the pressure distribution is shown in Fig. 16. As expected, the second mode dominates during the linear full contact phase which terminates after $1.6 \mathrm{~s}$. Separation now occurs, but the two pressure peaks then gradually become unequal and eventually there is a transition to a state with a single contact region-in other words a first mode solution with separation. Notice that the final state preserves the usual quasi-antisymmetric property in that the hot spots are alternately located on the two opposing surfaces.

Similar results were obtained at speed $V_{2}$ which exceeds the critical speed for modes with wavelengths $L, L / 2$, and $L / 3$. The

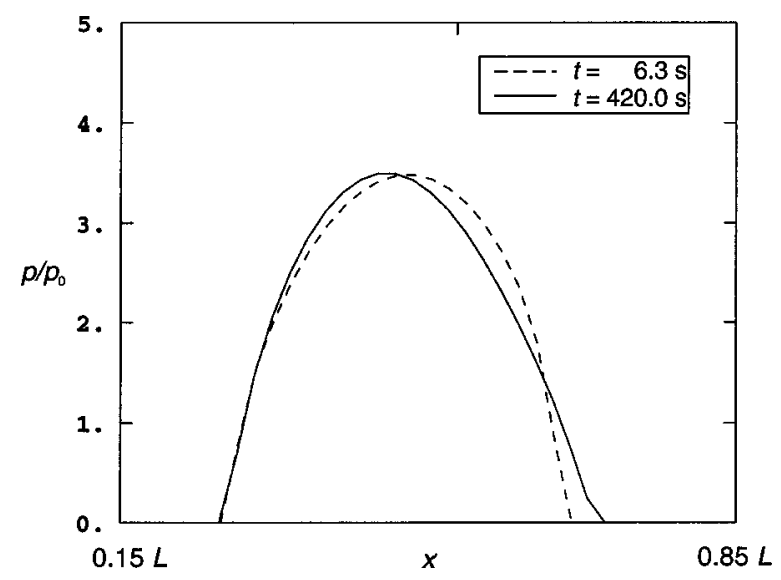

Fig. 14 Contact pressure distribution in the transient stage with contact separation and in the quasi-steady state

initial perturbation was dominated by the short wavelength perturbations, but once separation occurs, there is a gradual transition to a final steady state with a single contact region.

Simulations were also performed at speed $V_{3}$ which is above the critical speed for the second mode (wavelength $L / 2$ ) but below it for the first mode. As expected, only second mode disturbances were observed throughout the transient process, culminating in a steady state with two contact regions.

\section{Conclusions}

The two-dimensional transient simulation presented in this paper confirms the analytical predictions of Lee and Barber [4] and extends our knowledge of the TEI process into the nonlinear regime, where separation regions occur at the sliding interface. When only a single wavelength of perturbation is unstable, there is a transition to a steady state with the contact regions separated by the same wavelength, but the transient process is not monotonic. The migration speed in particular falls to significantly lower values than those obtained in the linear regime and then recovers to a steady state value. When several wavelengths are unstable, our results show that the final steady state is generally that corre-

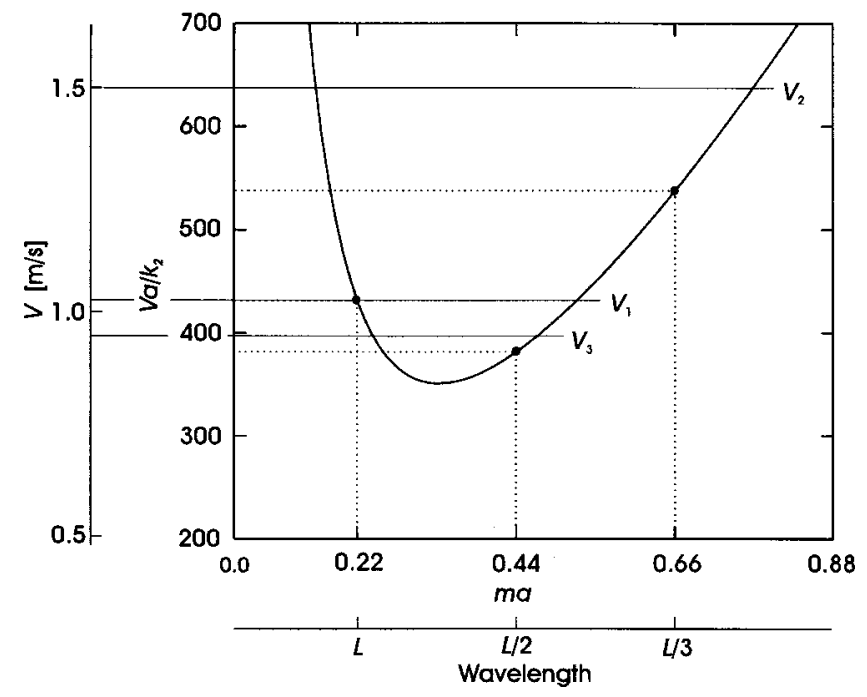

Fig. 15 Dimensionless critical speed determined by Lee and Barber's analytical model: $V_{1}, v_{2}$, and $V_{3}$-sliding speeds used in simulations 

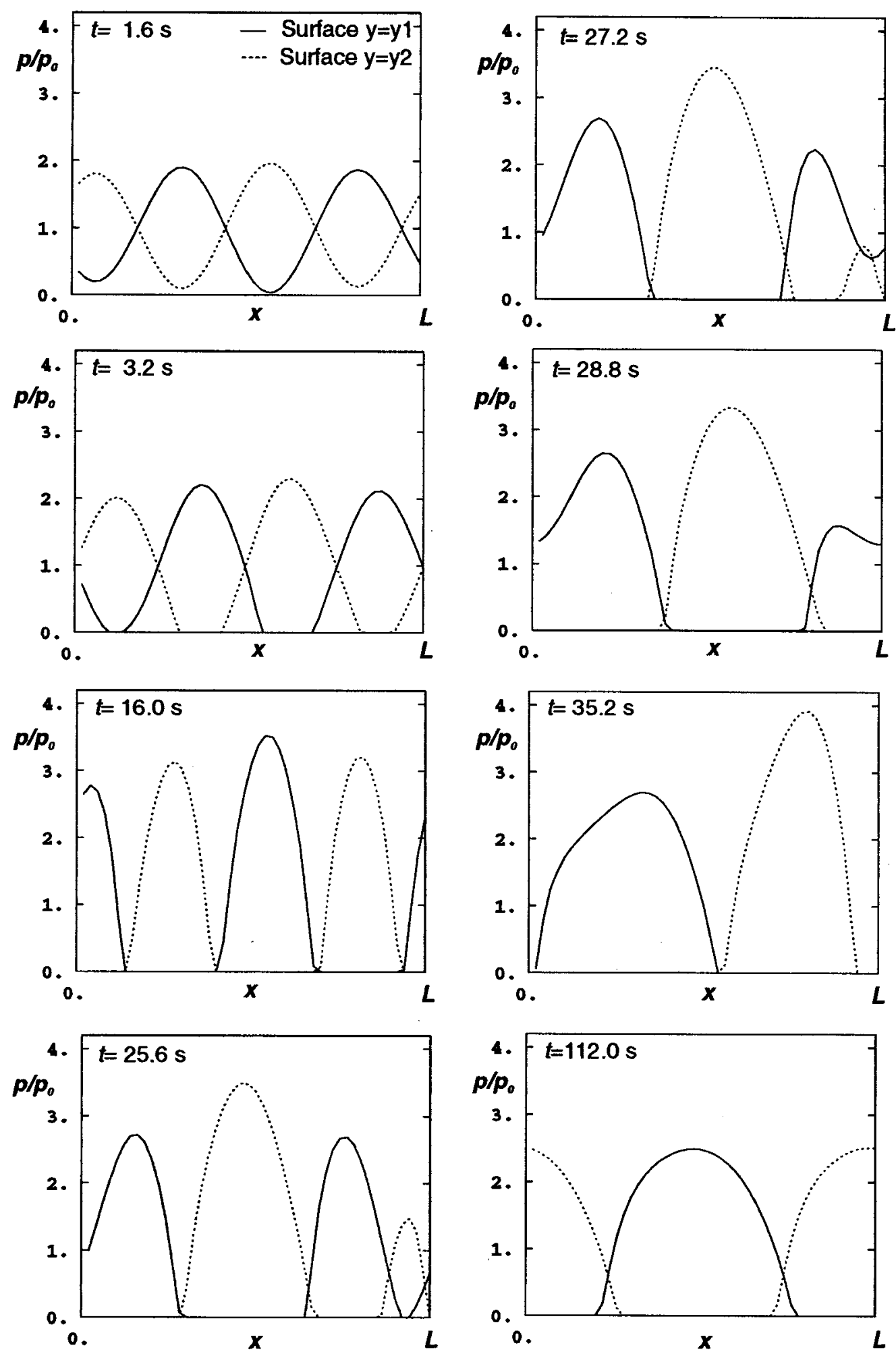

Fig. 16 Contact pressure distribution at a series of instants from simulation with two unstable modes

sponding to the longest unstable wavelength, even though other modes have more rapid growth rates in the linear regime.

\section{Nomenclature}

$$
\begin{aligned}
a & =\text { half-thickness of central layer } \\
A & =\text { dimensionless wavenumber } \\
b & =\text { growth rate }
\end{aligned}
$$

$$
\begin{aligned}
c & =\text { migration speed } \\
c_{p} & =\text { specific heat } \\
C & =\text { circumference of the disk } \\
\mathrm{Cu} & =\text { Courant number } \\
E & =\text { modulus of elasticity } \\
f & =\text { friction coefficient } \\
h & =\text { element length }
\end{aligned}
$$




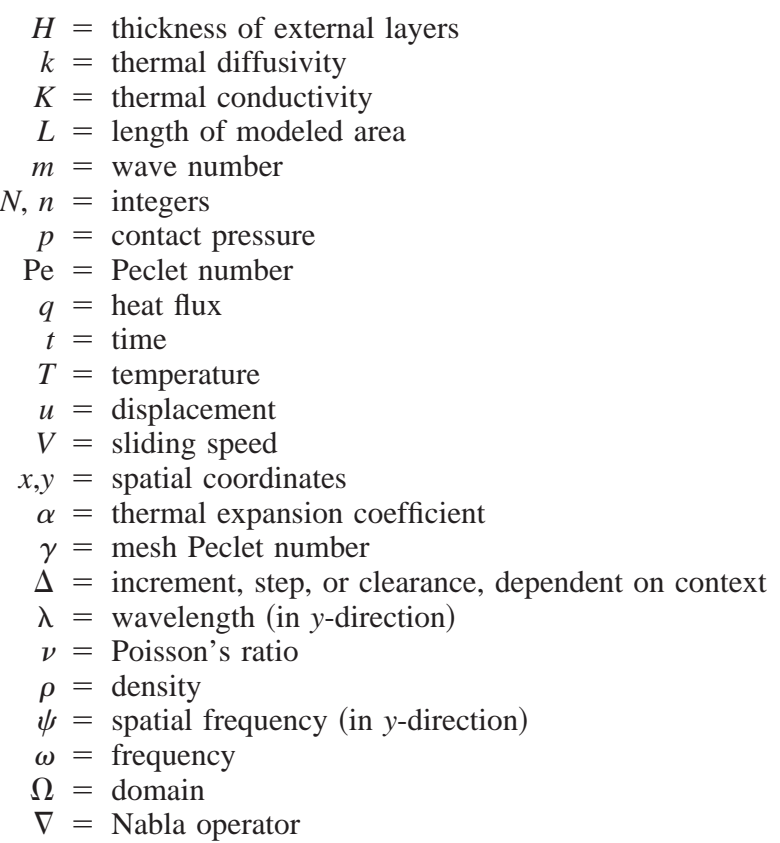

\section{Indices}

$a=$ amplitude

$m=$ mean

$c r=$ critical

$s=$ surface

\section{References}

[1] Inoue, H., 1986, "Analysis of Brake Judder Caused by Thermal Deformation of Brake Disk Rotors,'” SAE Paper 865131.

[2] Dow, T. A., and Burton, R. A., 1972, "Thermoelastic Instability of Sliding Contact in the Absence of Wear,', Wear, 19, pp. 315-328.
[3] Burton, R. A., Nerlikar, V., and Kilaparti, S. R., 1973, “"Thermoelastic Instability in a Seal-like Configuration,"' Wear, 24, pp. 177-188.

[4] Lee, K., and Barber, J. R., 1993, "Frictionally-Excited Thermoelastic Instability in Automotive Disk Brakes,', ASME J. Tribol., 115, pp. 607-614.

[5] Yi, Y.-B., Du, S., Barber, J. R., and Fash, J. W., 1999, "'Effect of Geometry on Thermoelastic Instability in Disk Brakes and Clutches,' ASME J. Tribol., 121, pp. 661-666.

[6] Barber, J. R., 1976, "Some Thermoelastic Contact Problems Involving Frictional Heating,’’ Q. J. Mech. Appl. Math., 29, pp. 1-13.

[7] Lee, K., and Dinwiddie, R. B., 1998, "Conditions of Frictional Contact in Disk Brakes and Their Effects on Brake Judder," SAE Paper 980598

[8] Kennedy, F. E., and Ling, F. F., 1974, "AA Thermal, Thermoelastic, and Wear Simulation of a High-Energy Sliding Contact Problem,' ASME J. Lubr. Technol., 97, pp. 497-508.

[9] Azarkhin, A., and Barber, J. R., 1985, "Transient Thermoelastic Contact Problem of Two Sliding Half-planes,' Wear, 102, pp. 1-13.

[10] Azarkhin, A., and Barber, J. R., 1986, "Thermoelastic Instability for the Transient Contact Problem of Two Sliding Half-Planes," ASME J. Appl. Mech., 53, pp. 565-572.

[11] Zagrodzki, P., 1990, "Analysis of Thermomechanical Phenomena in Multidisk Clutches and Brakes," Wear, 140, pp. 291-308.

[12] Heinrich, J. C., Huyakorn, P. S., Zienkiewicz, O. C., and Mitchell, A. R., 1977, "An "Upwind" Finite Element Scheme for Two-Dimensional Convective Transport Equation,' Int. J. Numer. Methods Eng., 11, pp. 131-143.

[13] Yu, C.-C., and Heinrich, J. C., 1986, "Petrov-Galerkin Methods for the TimeDependent Convective Transport Equation,'” Int. J. Numer. Methods Eng., 23, pp. 883-901.

[14] Yu, C.-C., and Heinrich, J. C., 1987, “'Petrov-Galerkin Method for Multidimensional, Time-Dependent, Convective-Diffusion Equations,' Int. J. Numer. Methods Eng., 24, pp. 2201-2215.

[15] ABAQUS, 1998, Theory Manual, Version 5.8, HKS.

[16] Craslaw, H. S., and Jaeger, J. C., 1959, Conduction of Heat in Solids, Clarendon Press, Oxford.

[17] Banaszek, J., 1993, “Fourier Mode Analysis in Error Estimation for FiniteElement Solution to Convective-Diffusive Phenomena," Arch. Thermodyn. 14, pp. 67-92.

[18] Lee, K., and Barber, J. R., 1993, "The Effect of Shear Tractions on Frictionally-Excited Thermoelastic Instability," Wear, 160, pp. 237-242.

[19] Anderson, A. E., and Knapp, R. A., 1990, "Hot Spotting in Automotive Friction Systems,"' Wear, 135, pp. 319-337.

[20] Yi, Y.-B., Barber, J. R., and Zagrodzki, P., 2000, “Eigenvalue Solution of Thermoelastic Instability Problems Using Fourier Reduction,' Proc. R. Soc. London, Ser. A, 456, No. 2003, pp. 2799-2821. 